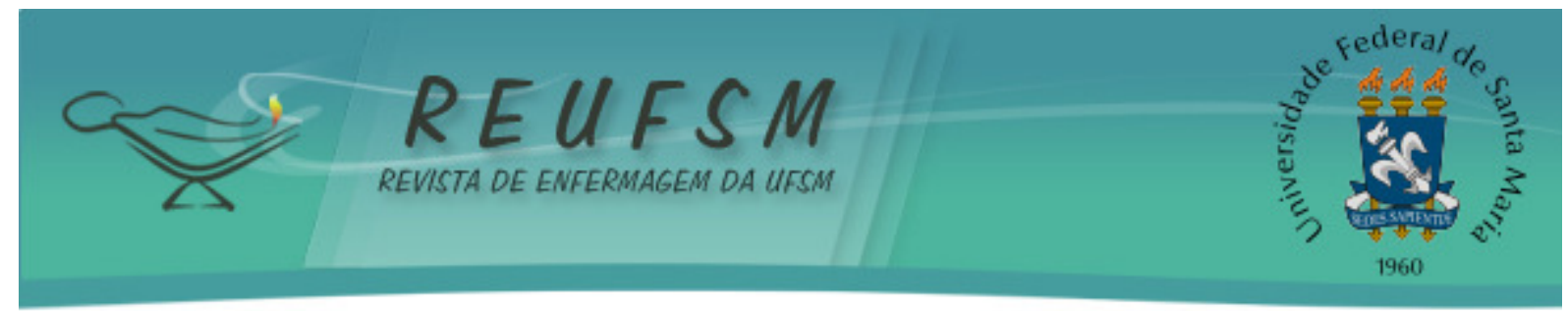

ARTIGO DE REFLEXÃO

\title{
PLANO DE PARTO: FERRAMENTA PARA O EMPODERAMENTO DE MULHERES DURANTE A ASSISTÊNCIA DE ENFERMAGEM
}

\section{BIRTH PLAN: A TOOL FOR WOMEN EMPOWERMENT DURING THE NURSING CARE \\ PLAN DE PARTO: HERRAMIENTA PARA EMPODERAMIENTO DE LA MUJER DURANTE LA ATENCIÓN DE LA ENFERMERÍA}

\author{
Adaiele Lucia Nogueira Vieira da Silva ${ }^{1}$ \\ Adriele Benites das Neves ${ }^{2}$ \\ Aniandra Karol Gonçalves Sgarbi ${ }^{3}$ \\ Rosely Almeida Souza ${ }^{4}$
}

Doi: $10.5902 / 2179769222531$

RESUMO: Objetivo: refletir sobre a importância do plano de parto na assistência de enfermagem, visando à autonomia da mulher. Método: trata-se de um texto reflexivo sobre o uso do Plano de Parto durante a assistência de enfermagem e o empoderamento da mulher. Resultados: enquanto ferramenta, o plano de parto centraliza o direito à informação e à decisão na mulher, tornando-a protagonista de seu próprio parto. Assim, garante-se o respeito ao princípio bioético de autonomia e às decisões da mulher,contribuindo para seu empoderamento no processo de gerar e parir. Considerações finais: evidencia-se, nesse estudo, a importância do uso do plano de parto pela enfermagem como instrumento de empoderamento da mulher. Ele é uma ferramenta que deve ser utilizada, visando à garantia de uma assistência de enfermagem humanizada, de qualidade e isenta de danos à mulher.

Descritores: Gravidez; Parto humanizado; Enfermagem obstétrica; Cuidados de enfermagem.

ABSTRACT: Aim: to reflect upon the importance of the birth plan in nursing care, aiming at women's autonomy. Method: This is a reflective text about the use of the Birth Plan during nursing care and also about women empowerment. Results: The birth plan centralizes women's right to information and to her decision-making, which makes her the protagonist of her own childbirth. Thus, it provides respect to the bioethical principle of autonomy and to women's decisions, contributing to their empowerment in the process of getting pregnant and giving birth. Final considerations: In this study, the importance of using the birth plan by the nursing staff as an instrument of women's empowerment is evidenced. The birth plan is a tool that should be used, aiming at the quality assurance of a harmless and humanized nursing care for women.

Descriptors: Pregnancy; Humanized birth; Obstetric nursing; Nursing care.

RESUMEN: Objetivo:reflexionar sobre la importancia del plan de parto en la prestación de cuidados de enfermería, objetivando la autonomía de la mujer. Método:Se trata de un texto reflexivo sobre la utilización del plan de parto durante los cuidados de enfermería y

\footnotetext{
${ }^{1}$ Enfermeira. Doutoranda em Saúde e Desenvolvimento na Região Centro-Oeste pela Universidade Federal de Mato Grosso do Sul. Campo Grande, Mato Grosso do Sul, Brasil. E-mail: adaiele@hotmail.com

2 Enfermeira. Especialização em Enfermagem Obstétrica E Ginecológica pela Faculdade de Ampére. Campo Grande, Mato Grosso do Sul, Brasil. E-mail: adrielebenites@yahoo.com.br

${ }^{3}$ Enfermeira. Doutoranda em Saúde e Desenvolvimento na Região Centro Oeste pela Universidade Federal de Mato Grosso do Sul. Campo Grande, Mato Grosso do Sul, Brasil. E-mail: ani_karol@yahoo.com.br.

${ }^{4}$ Enfermeira. Doutoranda em Saúde e Desenvolvimento na Região Centro Oeste pela Universidade Federal de Mato Grosso do Sul. Campo Grande, Mato Grosso do Sul, Brasil. E-mail: roselyalmeida@usp.br
} 


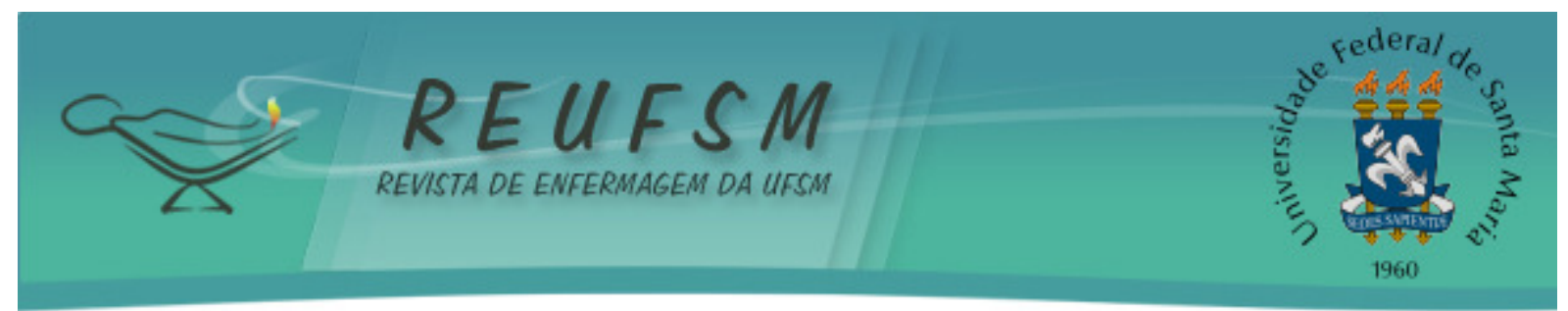

el empoderamiento de la mujer. Resultados:Como herramienta, el plan de parto se centra en el derecho de las mujeres a la información y a la tomada de decisiones como protagonista de su propio parto. Por este motivo, el respeto de los principios bioéticos de autonomía y decisiones de las mujeres está garantizado, contribuyendo para la potenciación de su papel en el proceso de generar y dar la luz.Consideraciones finales:Se destaca, en este estudio, la importancia de utilizar el plan de parto como un instrumento de potencialización de la mujer. Este es una herramienta que debe ser utilizado, a fin de garantizar una atención de enfermería de calidad, humana y libre de daños a la mujer.

Descriptores: Embarazo; Parto humanizado; Enfermería obstetricia; Los cuidados de enfermería.

\section{INTRODUÇÃO}

A gestação e o parto são eventos naturais e fisiológicos que, por sua vez, são determinados por processos individuais e sociais. Além disso, consistem numa experiência humana acrescida de valores, crenças, expectativas e preocupações que são dependentes da qualidade e quantidades das informações disponibilizadas a essas mulheres. ${ }^{1-6}$

Nesse contexto, é determinante a atuação dos profissionais da área de saúde, principalmente do enfermeiro, que por meio do acolhimento terá a oportunidade de promover uma escuta qualificada a cada mulher, para que esta se empodere de conhecimento e participe com autonomia de todas as decisões que envolvam o processo de gravidez e parto. ${ }^{2-6}$ Aliás, é oportuno mencionar que o pré-natal de risco habitual pode ser realizado pelo profissional enfermeiro. ${ }^{7}$

A atuação do enfermeiro na consulta de pré-natal é de suma importância para a garantia de uma assistência integral e de qualidade. É por meio desta que se pode garantir a extensão da cobertura, além de subsidiar a construção de relações de comunicação, que serão úteis para o acompanhamento e o acolhimento dessa gestante, em todas as fases do seu processo de gestar e parir. ${ }^{8}$

Durante a consulta de enfermagem, o enfermeiro pode fornecer orientações e informações, visando o empoderamentodessa mulher e contribuindo para que se torne mais ativa durante todo o processo. ${ }^{4} \mathrm{Um}$ instrumento que pode subsidiar esta prática é o Plano de Parto (PP), pois oferece um detalhamento das preferências e expectativas relacionadas ao processo gravídico e puerperal, podendo ser valioso como uma ferramenta de educação e comunicação. Os elementos mais importantes de um PP incluem o manejo da dor, medidas de conforto, as preferências pós-parto, o local do parto e reflexões sobre as crenças. ${ }^{3}$

Neste cenário, o interesse pela temática surgiu durante a experiência profissional no cuidado prestado no pré-natal, parto, pós-parto e puerpério. Na prática assistencial, foi possível observar que, apesar de toda uma série de políticas que buscam a qualidade e a humanização do processo gestar e parir, ainda impera, no Brasil, a prática do modelo tradicional, hospitalocêntrico e centrado no cuidado médico e não na mulher. ${ }^{9}$ Assim, este artigo de reflexão é oriundo de um Trabalho de Conclusão de Curso de pós-graduação em Enfermagem Obstétrica e Ginecológica, que perante uma lacuna de conhecimento acerca do Plano do Parto e da assistência de enfermagem, buscou discutira importância do Plano de Parto na assistência de enfermagem, visando o empoderamento da mulher. 


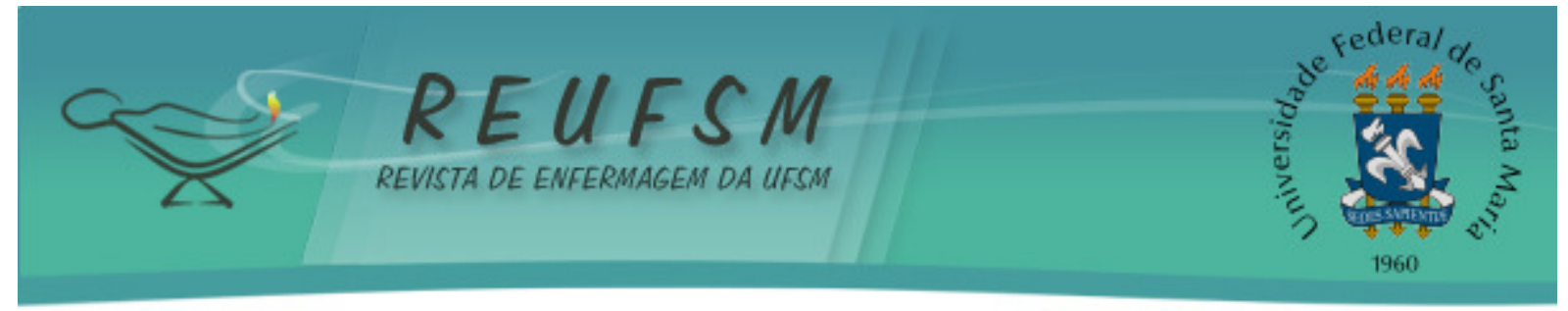

METÓDO

Para fundamentar a reflexão,realizou-se a buscade artigos científicos publicados em periódicos nacionais e internacionais, no idioma português, inglês ou espanhol.

A coleta de dados ocorreu nas bases de dados Medical Literature Analysis and Retrival System Online (MEDLINE), Base de Dados de Enfermagem (BDENF), Literatura Latino-Americana e do Caribe em Ciências da Saúde (LILACS), National Library of Medicine (PUBMED), Cochrane Library e na biblioteca eletrônica Scientific Electronic Library Online (SciELO). Para tal, utilizou-se os termos "plano de parto" e "assistência de enfermagem", acrescido da expressão booleano and, seguidos pelos descritores "cuidados de enfermagem" and "parto humanizado".

Foram selecionados artigos publicados nos últimos 10 anos, entre 2006 e 2016, cujorecorte temporal foi considerado uma vez que a temática começou a ser discutida com mais ênfase a partir do primeiro documento oficial publicado em 1996 pela Organização Mundial da Saúde (OMS). No entanto, somente a partir de 2006 foi possível encontrar mais produções acerca desse tema, como também não foi possível um recorte mais atual nos últimos cinco anos, pois não dispunha de publicações suficientes que atendessem aos objetivos propostos.

Os critérios de inclusão foram: produções nacionais e internacionais; objeto de estudona área de assistência de enfermagem no pré-natal e a construção do plano de parto; produções nos idiomas português, inglês ou espanhol, com textos completos de disponibilidade pública. Excluiu-se artigos repetidos em mais de uma base de dados, os editoriais e os estudos que, apesar de apresentarem os descritores selecionados, não possuíam relação com o objetivo proposto.

\section{RESULTADOS E DISCUSSÃO}

\section{Plano de parto e empoderamento}

Dentre as principais ferramentas para o empoderamento da mulher no processo de gestar e parir, destaca-se o PP, que pode ser elaborado e planejado a partir das demandas da mulher com o auxílio dos profissionais da área de saúde, principalmente o enfermeiro. ${ }^{2}$ O conceito de Plano de Parto e Nascimento foi descrito por Sheila Kitzinger, em 1980, nos Estados Unidos. ${ }^{10}$ Trata-se de um documento de caráter legal, contendo as escolhas da mulher para o seu pré-parto, parto e pós-parto. ${ }^{11}$

No ano de 1996, a OMS publicou o documento "Care in Normal Birth: a practical guide", que preconizava o uso de boas práticas na assistência obstétrica. 0 primeiro item desse documento evidenciava o PP como uma estratégia útil e que deveria ser estimulada durante a assistência prestada no pré-natal. 0 plano precisa estar disponível ainda no início do trabalho de parto. É elaborado com o consentimento e aceitação da mulher e também pode ser acordado com os familiares. ${ }^{12}$

O PP tem como objetivo orientar e preparar não apenas a mulher e seu respectivo cônjuge, mas também a equipe de saúde que atenderá o parto. Além disso, precisa evidenciar os procedimentos que geram conforto à parturiente e aqueles indesejáveis, sendo que a mulher também deve ser comunicada, caso ocorra qualquer alteração que necessite de intervenção. ${ }^{12}$

Entre as maneiras possíveis para a sua elaboração, a forma de carta é a mais utilizada e deve trazer as preferências da gestante de forma clara para orientar os profissionais de saúde que acompanharão o processo. 0 texto precisa ser elaborado durante o acompanhamento pré-natal e pode conter orientações sobre alimentação, exercícios físicos e respiratórios preparatórios. ${ }^{10-12}$ 


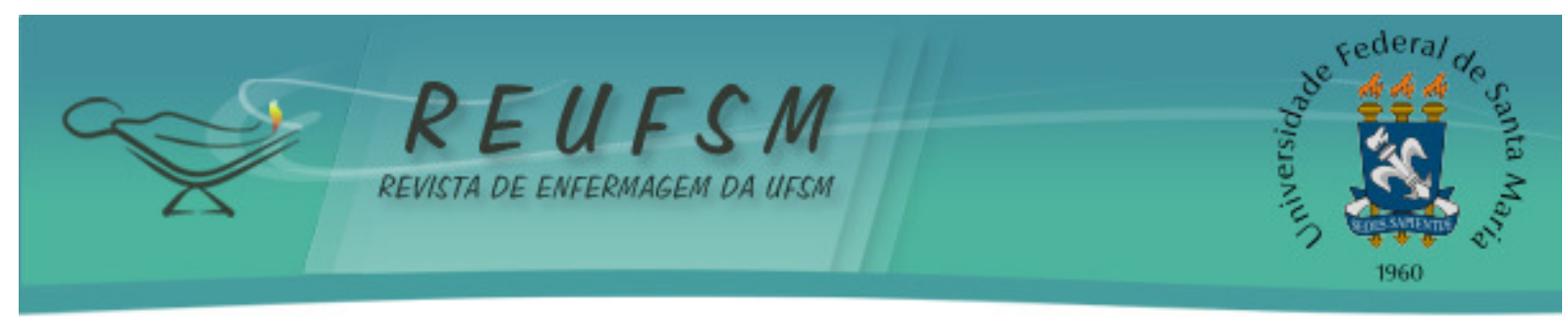

Enquanto ferramenta, o PP centraliza o direito à informação e à decisão na mulher, tornando-a protagonista de seu próprio parto, assim como os seus familiares. ${ }^{12}$ Logo, quando se remete à importância do PP, concomitantemente se fala do respeito ao Princípio Bioético da Autonomia, o qual se refere à autodeterminação, direito de liberdade, privacidade, escolha individual e livre vontade. ${ }^{13} 0$ respeito a esse princípio subsidia o controle da mulher sobre o seu processo parturitivo, proporcionando a esta a satisfação em poder decidir, além de minimizar os possíveis medos que emanam do processo de parir. ${ }^{10-12}$

A literatura aponta o PP como um componente educativo de alto potencial, uma vez que tem a capacidade de melhorar a comunicação entre os profissionais envolvidos nessa assistência e a usuária. ${ }^{10-12}$ No que tange ao seu caráter educativo, o PP possibilita à mulher o acesso a informações, subsidiando a construção de conhecimentos indispensáveis para a tomada de decisão sobre o parto e o nascimento do seu filho.

Em um estudo desenvolvido na Espanha, o PP foi denominado de Plano de Parto e Nascimento e considerado representante ímpar do Sistema Nacional de Saúde da Espanha. No estudo em questão, os autores afirmaram que este plano influenciava de forma benéfica o trabalho de parto e sua finalização, aumentando as dimensões de segurança, eficácia e satisfação das mulheres, assim como seu empoderamento. ${ }^{11}$

É oportuno mencionar que, no planejamento do parto, a aliança terapêutica estabelecida entre a parturiente e o enfermeiro possibilita a criação de vínculos e podeauxiliar na preferênciada mulher quanto à via, serviço e profissionais que realizarão a assistência ao parto. Não obstante, também subsidia a escolha de procedimentos no momento do parto tanto em situações em que este decorre sem intercorrências como em caso de possíveis complicações. ${ }^{14}$

Estudos internacionais apontaram a eficácia do PP, com o uso crescente nos serviços de saúde, que prestam cuidados especializados no pré-natal, parto e no puerpério. ${ }^{15-17}$ Uma pesquisa desenvolvida nos Estados Unidos com 109 mulheres que elaboraram o PP, constatou que as participantes se sentiram com maior controle e com uma visão mais clara sobre todo o processo de parturição, corroborando com os altos índices de satisfação frente às expectativas e à experiência vivenciada durante o parto. ${ }^{18}$ Diante da importância do PP para a autonomia da gestante/parturiente, é fundamental que a enfermagem conheça e se aproprie dessa ferramenta para aprimorar a assistência prestada, iniciando a elaboração do PP durante o pré-natal.

No Brasil, um estudo realizado em Londrina, Paraná, com um grupo de gestantes no último trimestre de gestação, acompanhadas por médicos tanto do setor público como do privado, tinha como objetivo explorar a necessidade de cuidado e participação dessas gestantes nas decisões acerca do parto. No decorrer da entrevista, foram indagadas sobre a importância do PP, e elas relataram que por meio deste têm acesso às informações sobre a gestação, os procedimentos a serem realizados no pré-parto, trabalho de parto e parto, conferindo à equipe profissional esta responsabilidade, além de requerer uma atenção obstétrica com profissionais competentes que ofereçam segurança e tranquilidade no momento do parto. ${ }^{19}$

Estudo realizado em Sorocaba, São Paulo, objetivou incentivar a participação materna na compreensão dos procedimentos hospitalares, por meio do PP, bem como a sua percepção da assistência ao parto. Foram empregadas ações educativas durante a elaboração do PP. Ao final do estudo, as participantes apontaram que a construção do PP se mostrou válida e importante, podendo ser um fator de satisfação no processo de parturição. ${ }^{20}$

Outro artigo acerca da violência obstétrica ponderou que refletir e escrever o PP não garante o cumprimento dos desejos da gestante pelo serviço de atenção ao parto, ou 


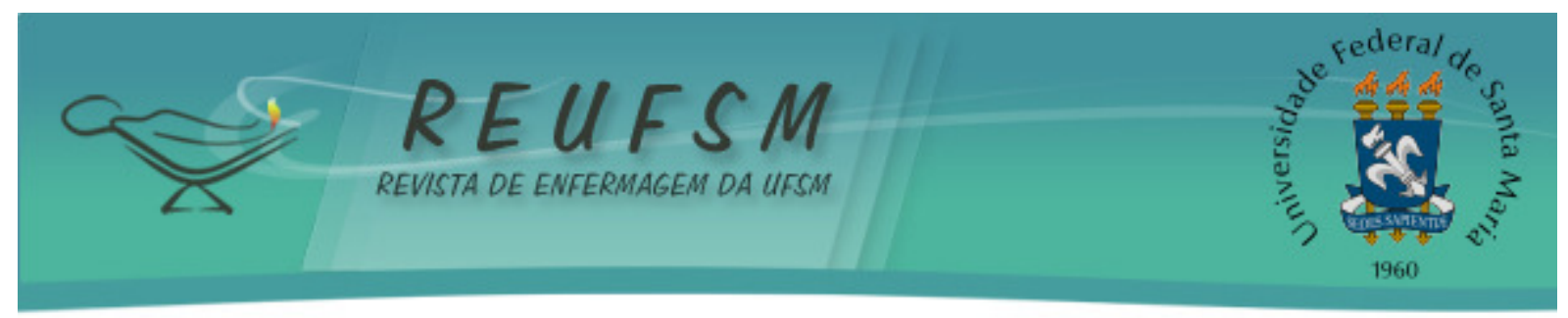

seja, é presumível que a reação dos profissionais não seja receptiva. Aponta que o descumprimento do PP, pelos profissionais e instituições, pode ser ocasionado pelo aumento da tensão e de conflitos entre provedores e mulheres e a criação de um ambiente de desconfiança entre os envolvidos. Para os autores, a elaboração de PP precisa ser estimulada por profissionais que acreditam nas evidências científicas e nos direitos das mulheres, para que a proposta do PP não seja transformada em mais um formulário a ser preenchido de maneira burocrática e sem valor. ${ }^{21}$

\section{A enfermagem e a construção do plano de parto}

A assistência de enfermagem prestada durante o pré-natal, além de contribuir com a redução da mortalidade materna, garante a detecção precoce das complicações próprias da gestação e o tratamento adequado de doenças maternas pré-existentes. Ainda, o acolhimento e a escuta realizada nesse momento são itens essenciais na política de humanização e, quando realizados adequadamente, corroboram para o fortalecimento de vínculos e para a continuidade dos cuidados.

Apesar das evidências da importância do PP como instrumento para fortalecer a autonomia da gestante, no município de Belo Horizonte foi realizado estudo que apontou que muitos profissionais de saúde não estão utilizando ou orientando as mulheres sobre o PP. Mesmo a cartilha da gestante fazendo menção à sua elaboração, constatou-se que $75 \%$ mulheres desconheciam essa ferramenta. ${ }^{22}$

A importância do PP também é evidenciada em um estudo realizado no Egito, com 260 mulheres, as quais foram divididas em dois grupos: o grupo controle, formado por 130 gestantes que receberam cuidados de rotina preconizados pela instituição; e o grupo de intervenção, composto também por 130 mulheres, que além dos cuidados de rotina, foram instruídas e acompanhadas durante todo o processo de elaboração do PP. Este estudo apontou que, além do aumento no grau de satisfação das parturientes que possuíam um $\mathrm{PP}$, elas apresentaram uma diminuição significativa no nível de dor e os recém-nascidos deste grupo tiveram as melhores pontuações na Escala de Ápgar. Desse modo, os autores recomendaram o uso do PP não apenas para aumentar o nível de satisfação das mulheres, mas também para a melhora dos resultados maternos e neonatais. ${ }^{23}$

Por fim, o poder libertador do saber e do cuidado de enfermagem permitem outras possibilidades à mulher, além de promover a utilização e desenvolvimento das capacidades existentes. No que se refere ao empoderamento, este se torna "libertador à medida em que os cuidados de enfermagem a incentivam para aquilo que são capazes, ou seja, promovendo a autonomia da mulher". 6:427

\section{CONSIDERAÇÕES FINAIS}

Os estudos apontaram a importância da utilização do PP para o empoderamento da mulher no pré-natal, parto e puerpério, garantindo, assim, o respeito ao Princípio Bioético da autonomia nesse período. Logo, o uso dessa ferramenta pode garantir uma melhor qualidade da assistência para o binômio mãe e filho, contribuindo para a redução e a eliminação de atos de negligência, imperícia e imprudência, assim como pode promover a emancipação da mulher. Além disso, também pode fortalecer o laço afetivo quando esta delega ou compartilha as decisões relacionadas ao processo de parturição com amigos, familiares e companheiro.

Além disso, a reflexão contribui para justificar o uso do PP pelos profissionais da área de saúde, principalmente o enfermeiro, que poderão utilizá-lo como estratégia para substanciar sua prática do cuidado. Além disso, salienta-se a necessidade de estudos, 


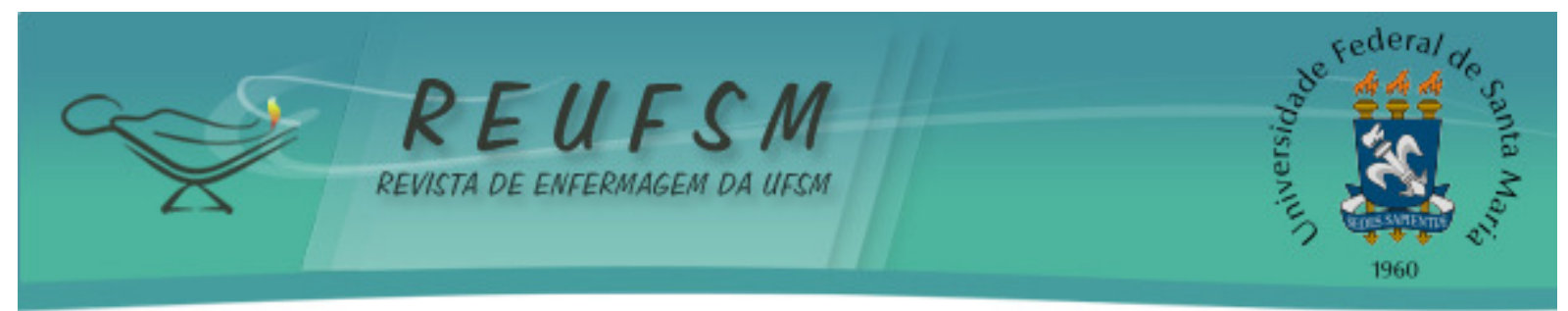

pautados na autonomia do enfermeiro e de outros profissionais envolvidos no processo de parturição, sendo estes agentes fomentadores da conquista da autonomia da mulher durante todo o gestar e parir, tornando-a protagonista de seu próprio parto.

Ressalta-se a escassez de publicações nacionais sobre o PP como ferramenta utilizada durante a assistência de enfermagem, evidenciando a necessidade de novas pesquisas que apresentem resultados e reflexões sobre a importância da temática tanto para o fortalecimento da categoria profissional como para a prática assistencial da enfermagem na área da saúde da mulher.

\section{REFERÊNCIAS}

1. Silva SPC, Prates RCG, Campelo BQA. Parto normal ou cesariana? Fatores que influenciam na escolha da gestante. Rev Enferm UFSM [Internet]. 2014 jan-mar [acesso em $2016 \quad \mathrm{abr} \quad 15] ; 4(1): 1-9$

http: //periodicos.ufsm.br/reufsm/article/view/8861/pdf. doi:http://dx.doi.org/10.5902/217976928861.

2. Pereira ALF, Bento AD. Autonomia no parto normal na perspectiva das mulheres atendidas na casa de parto. Rev RENE. 2011;12(3):471-7.

3. Aragon M, Chhoa E, Dayan R, Lohn Z, Buhler K. Perspetives of expectante women and health care providers on birth plans. J ObstetGynaecol Can. 2013;35(11):979-85.

4. Melo KL, Vieira BD, Alves VH, Rodrigues DP, Leão DCMR, Silva LA. O comportamento expresso pela parturiente durante o trabalho de parto: reflexos da assistência do prénatal. RevPesquiCuid Fundam (Online) [Internet]. 2014 jul-set [acesso em 2016 abr 15];6(3):1007-20. Disponível

em: http://www.seer.unirio.br/index.php/cuidadofundamental/article/view/3124.

5. Malheiros PA, Alves VH, Rangel TSA, Vargens OMC. Labor and birth: knowledge and humanized practices. Texto \& Contexto Enferm [Internet]. 2012 abr-jun [acesso em 2016 jun 17];2012;21(2):329-37. Disponível em: http: / / www.scielo.br/scielo.php?script=sci_arttext\&pid=S0104-07072012000200010.

6. Caires TLG, Santos RS. O saber da enfermagem obstétrica e suas contribuições sociais para a autonomia da parturiente. Rev Enf Profissional. 2014;1(2):422-35.

7. Brasil. Lei n. 7.498 de 25 de junho de 1986. Dispõe sobre a regulamentação do exercício da enfermagem, e dá outras providências. Diário Oficial da União, Brasília [Internet]. 1986 jun 26 [acesso em 2016 maio 25]. Disponível em: http://www.planalto.gov.br/ccivil_03/leis/L7498.htm.

8. Araujo SM, Silva MSD, Moraes, RC, Alves DS. A importância do pré-natal e a assistência de enfermagem. Veredas [Internet]. 2010 jul-dez [acesso em 2016 jun 15];3(2):61-7. Disponível

em: http: //veredas.favip.edu.br/ojs/index.php/veredas1/article/view/98/211.

9. Rede Parto do Princípio. Dossiê Violência Obstétrica: "Parirás com dor" [Internet]. 2012 [acesso em $2016 \quad$ abr 10$]$ 1 http://www.senado.gov.br/comissoes/documentos/SSCEPI/DOC\%20VCM\%20367.pdf.

10. Hasenmiller R. Do birth plans influence childbirth satisfaction? [Student projects] Philadelphia: Philadelphia University; 2001.

11. Suárez-Cortés M, Armero-Barranco D, Canteras-Jordana M, Martínez-Roche ME. Use and influence of delivery and birth plans in the humanizing delivery process. 


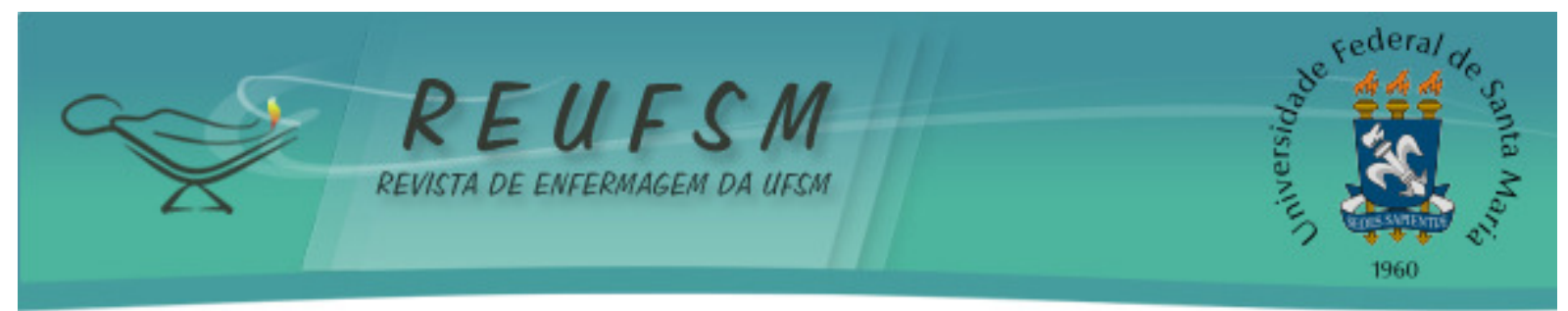

RevLatinoamEnferm (Online) [Internet]. 2015 maio-jun [acesso em 2016 abr 10];23(3):5206. Disponível em: http://www.scielo.br/scielo.php?script=sci_arttext\&pid=S0104$11692015000300520 \& \operatorname{lng}=$ en\&nrm=iso\&tlng=en\&ORIGINALLANG=en.

12. World Health Organization. Safe Motherhood Unit. Care In normal birth: a pratical guide. Genebra: OMS; 1996.

13. Beauchamp TL, Childress JF. Princípios de ética biomédica. $2^{\mathrm{a}}$. ed. São Paulo: Edições Loyola; 2011.

14. Campos AS, Almeida ACCH, Santos RP. Crenças, mitos e tabus de gestantes acerca do parto normal. Rev Enferm UFSM [Internet]. 2014 abr-maio [acesso em 2016 abr 10];4(2):332-41. Disponível

http://periodicos.ufsm.br/reufsm/article/view/10245/pdf.

15. Hidalgo-Lopezosa $P$, Rodríguez-Borrego MA, Muñoz-Villanueva MC. Are birth plans associated with improved maternal or neonatal outcomes? MCN Am J Matern Child Nurs. [Internet]. 2013 maio-jun [acesso em 2016 abr 18];38(3):150-6. Disponível em: http//www.ncbi.nlm.nih.gov/pubmed/23625102.

16. Hadar E, Raban O, Gal B, Yogev Y, Melamed N. Obstetrical outcome in women with self-prepared birth plan. J Matern Fetal Neonatal Med [Internet]. 2012 out [acesso em 2016 abr 18];25(10):2055-57. Disponivel em: http: / /www.ncbi.nlm.nih.gov/pubmed/22489709.

17. Magoma M, Requejo J, Campbell O, Cousens S, Merialdi M, Filippi V. The effectiveness of birth plans in increasing use of skilled care at delivery and postnatal care in rural Tanzania: a cluster randomised trial. Trop Med Int Health [Internet]. 2013 abr [acesso em 2016 abr 18];18(4):435-43. Disponivel em: http: //www.ncbi.nlm.nih.gov/pubmed/23383733.

18. Mei JY, Afshar Y, Gregory KD, Kilpatrick SJ, Esakoff TF. Birth plans: what matters for birth experience satisfaction. Birth [Internet]. 2016 jun [acesso em 2016 jun 18];43(2):14450. Disponivel em: http://www.ncbi.nlm.nih.gov/pubmed/26915304.

19. Sodré TM, Bonadio IC, Jesus MCP, Merighi MAB. Necessidade de cuidado e desejo de participação no parto de gestantes residentes em Londrina-PR. Texto \& Contexto Enferm [Internet]. 2010 set [acesso em 2016 nov 6];19(3):452-60. Disponível em: http://www.scielo.br/pdf/tce/v19n3/a06v19n3.pdf.

20. Almeida JM de, Guimarães VB, Martins CA. Planejamento do parto - instrumento educativo compartilhado com gestantes na UBS Jardim Simus. Revista da Faculdade de Ciências Médicas de Sorocaba [Internet]. 2015 out [acesso em 2016 nov 9];17 Supl (32 Congresso da SUMEP). Disponível em: http://revistas.pucsp.br/index.php/RFCMS/article/view/24863.

21.Tesser, C, Knobel R, Andrezzo, H, Diniz, S. Violência obstétrica e prevenção quaternária: o que é e o que fazer. Revista Brasileira de Medicina de Família e Comunidade [Internet]. 2015 jun [acesso em 2016 nov 9];10(35):1-12. Disponível em: https://rbmfc.org.br/rbmfc/article/view/1013.

22. Silva SG, Silva EL, Souza KV, Oliveira DCC. Perfil de gestantes participantes de rodas de conversa sobre o plano de parto. Enferm Obstétrica. 2015;2(1):9-14. 


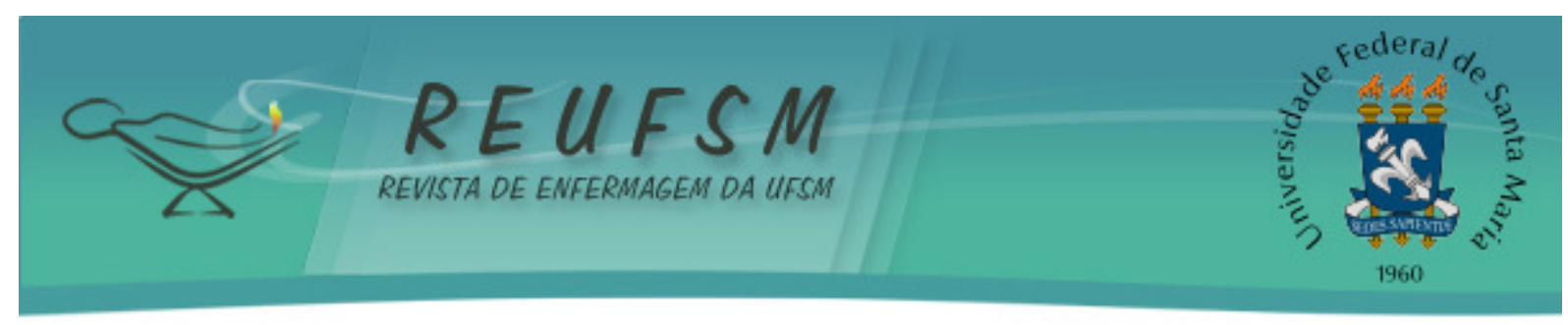

23. Hussain FA, Sayed MHE, Abd ES, El-Nemer A. Effect of implementing a birth plan on womens' childbirth experiences and maternal \& neonatal outcomes. J Edc Pract. 2015;6(6):24-32.

Data de recebimento: 07/06/2016

Data de aceite: 04/01/2017

Contato com autor responsável: Adaiele Lucia Nogueira Vieira da Silva

Endereço postal: Rua das Oliveiras, 139, Vila Adelina. Cep: 79070-308. Campo Grande, Mato Grosso do Sul. E-mail: adaiele@hotmail.com 